\title{
Correlation of Surgical Pleth Index with Stress Hormones during Propofol-Remifentanil Anaesthesia
}

\author{
Xinzhong Chen, ${ }^{1,2}$ Carsten Thee, ${ }^{2}$ Matthias Gruenewald, ${ }^{2}$ Christoph Ilies, ${ }^{2}$ Jan Höcker, ${ }^{2}$ \\ Robert Hanss, ${ }^{2}$ Markus Steinfath, ${ }^{2}$ and Berthold Bein ${ }^{2}$ \\ ${ }^{1}$ Department of Anaesthesia, Women's Hospital, School of Medicine, Zhejiang University, Hangzhou 310058, China \\ ${ }^{2}$ Department of Anaesthesiology and Intensive Care Medicine, University Hospital Schleswig-Holstein, Campus Kiel, \\ Schleswig-Holstein, 24105 Kiel, Germany
}

Correspondence should be addressed to Xinzhong Chen, chenxinz@zju.edu.cn and Berthold Bein, bein@anaesthesie.uni-kiel.de

Received 25 June 2012; Accepted 18 July 2012

Academic Editors: N. J. Christensen and E. O. Martin

Copyright (c) 2012 Xinzhong Chen et al. This is an open access article distributed under the Creative Commons Attribution License, which permits unrestricted use, distribution, and reproduction in any medium, provided the original work is properly cited.

Eighty patients undergoing elective ear-nose-throat surgery were enrolled in the present study to investigate the relationship between surgical pleth index (SPI) and stress hormones (ACTH, cortisol, epinephrine, norepinephrine) during general anaesthesia which was induced and maintained with propofol and remifentanil using a target-controlled infusion. The study concluded that the SPI had moderate correlation to the stress hormones during general anaesthesia, but no correlation during consciousness. Furthermore, SPI values were able to predict ACTH values with high sensitivity and specificity.

\section{Introduction}

Excessive intraoperative stress evoked by surgical nociceptive stimulation may influence patients' outcome, length of hospital stay, and overall costs of hospital care $[1,2]$. To achieve adequate analgesia (antinociception) blunting the intraoperative stress response, most notably haemodynamic instability, it is crucial to use an ideal variable for assessing the stress level or, perhaps more accurately, the balance of nociception-antinociception [3], during general anaesthesia. Unfortunately, such an ideal (continuous, noninvasive, accurate) variable, which may be used for guiding administration of analgesics to attenuate the stress response, is still missing. Traditionally, clinical signs like somatic (movement) or autonomic (tachycardia, hypertension, sweating, and tearing) responses are used to evaluate whether analgesia is adequate, which has been proved to be unreliable demonstrating low specificity [4-7].

Recently, the so-called surgical pleth index (SPI), a novel multivariate index using two continuous cardiovascular variables, the normalized heart beat interval (HBI) and the normalized pulse wave amplitude (PPGA), was developed and proposed to quantify the intraoperative stress level or nociception during general anaesthesia [8-10]. Wennervirta et al. [9] found that the SPI performed better than heart rate, blood pressure, or response entropy in detecting nociceptive stimuli during general anaesthesia [11, 12]. Huiku et al. [8] and Gruenewald et al. [10] also showed that the SPI had a negative correlation with remifentanil effect-site concentration $\left(\mathrm{Ce}_{\text {remi }}\right)$ and a positive correlation with the intensity of stimulation during total intravenous anaesthesia with propofol and remifentanil. Moreover, one of our previous studies [13] suggested that SPI could be used for guiding remifentanil administration during propofol-remifentanil anaesthesia, which resulted in lower remifentanil consumption, more stable haemodynamics, and fewer unwanted events. Given the results from the above studies, the SPI seems like a relatively ideal variable for measurement of intraoperative stress or nociception.

Stress hormones such as cortisol, adrenocorticotropic hormone $(\mathrm{ACTH})$, epinephrine, and norepinephrine have been validated to evaluate the magnitude of the surgical stress response $[14,15]$. The measurement of the above circulating stress hormones, however, requires blood samples and laboratory analysis and is not suitable for bedside monitoring of intraoperative stress [16]. Owing to the 
absence of a gold standard for evaluating the performance of an indicator of intraoperative stress level, we used the circulating stress hormones as a tool to evaluate the SPI performance in measuring the stress level during general anaesthesia.

In the present prospective, single-blinded, randomized study, we investigated the relationship between SPI values, BIS values, arterial blood pressure (ABP), heart rate (HR) and stress hormones' values (ACTH, cortisol, epinephrine, and norepinephrine) at four event-related time points during propofol-remifentanil anaesthesia in patients undergoing ear-nose-throat (ENT) surgery. We hypothesized that (1) SPI has a different performance at different states of consciousness (loss of consciousness versus consciousness); (2) SPI has a better correlation with stress hormones than ABP, HR, or the BIS.

\section{Materials and Methods}

2.1. Patients and Study Design. After obtaining approval of the institutional review board of the University Hospital Schleswig-Holstein, Campus Kiel, and written informed consent, 80 patients ASA physical status I-II, aged 18-70 years, scheduled for elective ear-nose-throat (ENT) surgery expected to last at least $1 \mathrm{~h}$ were enrolled in this prospective, single-blinded, randomized trial. Patients with a history of CNS disease (e.g., neurological disorders, head injury, seizure disorders), chronic use of psychoactive medication or abuse of alcohol or illicit drugs, and any clinical significant cardiovascular, renal, hepatic, or endocrinologic disorders were excluded. Patients were randomly allocated to one of two groups: SPI group in which remifentanil titration was guided by SPI values during anaesthesia maintenance or Control group in which remifentanil titration was guided by standard traditional inadequate anaesthesia criteria (Table 1 [17-19]). Some results of this study have been published previously [13].

Patients in both groups were premedicated with 20 $30 \mathrm{mg}$ dipotassium clorazepate the evening before and 3.75$7.5 \mathrm{mg}$ midazolam orally 30 minutes before surgery. Surgery always started in the morning to avoid bias caused by the circadian rhythm of circulating stress hormones. On arrival in the operating theatre, an intravenous catheter was inserted into a larger forearm vein, and standard monitoring including noninvasive blood pressure (NIBP), 5-lead electrocardiogram (ECG), and pulse oximetry $\left(\mathrm{SpO}_{2}\right)(\mathrm{S} / 5$ Anaesthesia Monitor, GE Healthcare, Helsinki, Finland) was applied. All patients in both groups were monitored with BIS and SPI. After the skin of the forehead had been degreased with alcohol, BIS electrodes (BIS sensor; Aspect Medical Systems, Natick, MA, USA) were positioned as recommended by the manufacturer, and electrode impedance was kept below $7.5 \mathrm{~K} \Omega$ to ensure optimal contact. SPI monitoring shared the same sensor of pulse oximetry which was clamped on the index finger. The calculation of the SPI was done by $10 \mathrm{~s}$ intervals and described elsewhere [8]. During the whole period of anaesthesia, NIBP was monitored with an interval of 3 minutes, and SPI, BIS, $\mathrm{SpO}_{2}$, and ECG were monitored continuously.
TABLe 1: Criteria for inadequate anaesthesia and hypotension or bradycardia.

\begin{tabular}{cl}
\hline Inadequate anesthesia & \\
Hypertension & $\begin{array}{l}\text { Mean blood pressure }>120 \% \text { of baseline } \\
\text { or }>100 \mathrm{mmHg}\end{array}$ \\
Tachycardia & $\begin{array}{l}\text { Heart rate }>90 \text { beats } \cdot \mathrm{min}^{-1} \\
\text { Coughing, chewing, grimacing }\end{array}$ \\
Somatic arousal & $\begin{array}{l}\text { Purposeful movement } \\
\text { Mean blood pressure }<80 \% \text { of baseline or }\end{array}$ \\
Hypotension & $\begin{array}{l}<60 \mathrm{mmHg} \\
\text { Heart rate }<80 \% \text { of baseline or } \\
\text { Bradycardia }\end{array}$ \\
\hline
\end{tabular}

Criteria for inadequate anaesthesia were modified based on previous studies.

Patients in both groups received target-controlled infusion (TCI) of propofol with a target effect-site concentration $\left(C e_{\text {prop }}\right)$ of $4 \mu \mathrm{g} \cdot \mathrm{mL}^{-1}$ and TCI of remifentanil with target effect-site concentration $\left(\mathrm{Ce}_{\text {remi }}\right)$ of $4 \mathrm{ng} \cdot \mathrm{mL}^{-1}$ via targetcontrolled infusion pumps (TCI; Asena Alaris, Cardinal Health, Basingstoke, UK) and $0.6 \mathrm{mg} \cdot \mathrm{kg}^{-1}$ rocuronium before tracheal intubation. For propofol, the pharmacokinetic model of Schnider and colleagues was used [20] and for remifentanil, the pharmacokinetic model of Minto and colleagues [21]. After intubation, the lungs were ventilated to an end-tidal carbon dioxide $\left(\mathrm{ETCO}_{2}\right)$ concentration of $35(30-40) \mathrm{mmHg}$. Ce $\mathrm{e}_{\text {prop }}$ was adjusted stepwisely by $0.5 \mu \mathrm{g} \cdot \mathrm{mL}^{-1}$ with 4 -minute intervals to maintain BIS level between 40 and 60 (however $\mathrm{Ce}_{\text {prop }}$ was not reduced below $2 \mu \mathrm{g} \cdot \mathrm{mL}^{-1}$ ), whereas the $\mathrm{Ce}_{\text {remi }}$ was not adjusted until start of the surgical procedure.

During anaesthesia maintenance, all patients, irrespective of the group assignment, were kept at BIS level of 4060 by adjusting the $C e_{\text {prop }}$ stepwisely by $0.5 \mu \mathrm{g} \cdot \mathrm{mL}^{-1}$ with an interval of $4 \mathrm{~min}$.

In the Control group, the $\mathrm{Ce}_{\text {remi }}$ was adjusted based on traditional signs and symptoms of inadequate anesthesia. Specifically, inadequate anaesthesia was defined as presence of symptoms detailed in Table 1 [17-19] and was treated by increasing the $\mathrm{Ce}_{\text {remi }}$ by $1 \mathrm{ng} \cdot \mathrm{mL}^{-1}$ step wisely until the maximum allowed concentration of $10 \mathrm{ng} \cdot \mathrm{mL}^{-1}$. If this was judged insufficient, urapidil $10 \mathrm{mg}$ was given i.v. Hypotension was treated initially by speeding intravenous infusion, then $\mathrm{Ce}_{\text {remi }}$ was decreased by $1 \mathrm{ng} \cdot \mathrm{mL}^{-1}$ stepwisely until the minimum concentration of $4 \mathrm{ng} \cdot \mathrm{mL}^{-1}$, and finally, $0.5 \mathrm{ml}$ Akrinor (an intravenous vasopressor, AWD Pharma, Dresden, Germany; $1 \mathrm{~mL}$ contain $100 \mathrm{mg}$ cafedrine and $5 \mathrm{mg}$ theodrenaline) was given intravenously. Atropine $0.5 \mathrm{mg}$ was used for bradycardia.

In the SPI group, the $\mathrm{Ce}_{\text {remi }}$ was adjusted to keep the SPI values between 20 and 50 by increasing or decreasing remifentanil plasma concentration by $1 \mathrm{ng} \cdot \mathrm{mL}^{-1}$ step wisely $\left(\mathrm{Ce}_{\text {remi }}\right.$ range was limited between 4 and $\left.10 \mathrm{ng} \cdot \mathrm{mL}^{-1}\right)$. In case of $20<$ SPI $<50$, inadequate anesthesia was treated as follows: urapidil $10 \mathrm{mg}$ i.v. for hypertension; Akrinor $0.5 \mathrm{~mL}$ i.v. for hypotension; atropine $0.5 \mathrm{mg}$ i.v. for bradycardia. A rescue medication was allowed (propofol bolus of $0.5 \mathrm{mg} \cdot \mathrm{kg}^{-1}$ ) if somatic arousal or a somatic response 
occurred despite BIS and SPI values within the predefined range.

To facilitate rapid emergence from anesthesia, 15 minutes before the expected end of surgery, $\mathrm{PEC}_{\text {prop }}$ was reduced in all patients, and a BIS value of over 60 but below 65 was allowed, whereas the $\mathrm{PEC}_{\text {remi }}$ remained unchanged until the end of surgery. All patients received $0.1 \mathrm{mg} \cdot \mathrm{kg}^{-1}$ piritramide for postoperative analgesia. At the end of surgery which was defined as the final surgical suture, both propofol and remifentanil were stopped.

2.2. Blood Sampling and Stress Hormones Assay. In both groups, blood samples were taken at four event-related time points (Base: baseline before anaesthesia; Intu: after tracheal intubation; Max: at maximum surgical trauma, defined intraoperatively by the attending surgeon; After-Max: $15 \mathrm{~min}$ after the maximum surgical trauma) for measurement of ACTH, cortisol, epinephrine, and norepinephrine. Samples were immediately placed into iced water, cool-centrifuged within $15 \mathrm{~min}$, and stored at $-25^{\circ} \mathrm{C}$ until further analysis. An improved reversed phase high-performance liquid chromatography technique was used for analysis (autosampler: AS 2000, Merck Hitachi, Darmstadt, Germany; pump: L6200 Intelligent Pump, Merck Hitachi, Darmstadt, Germany; detector: ECD, Merck- LaChrom L 3500 A, Merck Hitachi, Darmstadt, Germany; Analytical Column for separation: RP 18- equilibrated and tested, Bio-Rad Diagnostics, München, Germany; software: D 7000- HPLC- System, Merck Hitachi, Darmstadt, Germany). Reference range of ACTH: 7.2$63.6 \mathrm{pg} / \mathrm{mL}$; cortisol: $6.2-19 \mu \mathrm{g} / \mathrm{dL}$; epinephrine: $<84 \mathrm{pg} / \mathrm{mL}$; norepinephrine: $<420 \mathrm{pg} / \mathrm{mL}$.

2.3. Statistical Analysis. All data are presented as mean and SD or median and range, as appropriate. GraphPad Prism (Version 5.0, GraphPad Software Inc., San Diego, CA, USA) was used for statistical analysis. For numerical data, statistical analysis was performed with Student's $t$-test (for normally distributed data) and Mann-Whitney $U$ test (for nonnormally distributed data) or one-way analysis of variance with Student-Newman-Keuls test (for multiple comparisons); for nominal data statistical analysis was performed by means of a chi-square test. Correlation analysis between variables was performed with Spearman's rank correlation coefficient, and linear regression slopes were analyzed with $F$-test.

Receiver operator characteristics (ROCs) analysis was performed to evaluate and visualize whether SPI was able to indicate a predefined level of a stress hormone blood concentration (we defined the average level of a stress hormone at the time point "Base" as this specific level, because no well-accepted standard of stress hormone exists for denoting a stress state so far, and at the time point "Base" all patients were conscious and could be considered as experiencing kind of stress before induction of anaesthesia). Further, ROC analysis was used to depict threshold values for SPI to indicate the specific hormone level, based on pooled data.

A $P$ value of $<0.05$ was considered as statistically significant.
TABle 2: Demographic data.

\begin{tabular}{lccc}
\hline & SPI $(n=40)$ & Control $(n=40)$ & $P$ values \\
\hline Age (years) & $47 \pm 17$ & $46 \pm 17$ & 0.839 \\
Height $(\mathrm{cm})$ & $173 \pm 18$ & $171 \pm 93$ & 0.657 \\
Weight $(\mathrm{Kg})$ & $78 \pm 12$ & $75 \pm 17$ & 0.438 \\
Gender-M/F (n) & $13 / 27$ & $21 / 19$ & 0.254 \\
ASA I/II (n) & $18 / 22$ & $19 / 21$ & 1.000 \\
$\begin{array}{l}\text { Duration of } \\
\text { anesthesia (min) }\end{array}$ & $152 \pm 67$ & $173 \pm 84$ & 0.076 \\
$\begin{array}{l}\text { Duration of surgery } \\
\text { (min) }\end{array}$ & $109 \pm 61$ & $132 \pm 81$ & 0.108 \\
$\begin{array}{l}\text { Intubation to surgery } \\
\text { beginning (min) }\end{array}$ & $24 \pm 8$ & $25 \pm 10$ & 0.534 \\
\hline
\end{tabular}

Values are mean \pm SD or absolute numbers. No difference between groups. SPI: the group in which remifentanil administration was guided by SPI.

Control: the group in which remifentanil administration was guided by standard traditional inadequate anaesthesia criteria.

\section{Results}

All of the 80 patients finished the study and were included into final analysis for demographic data and clinical data such as duration of surgery and duration of anaesthesia. There were no differences between groups with regard to sex, age, height, body weight, duration of surgery, and duration of anaesthesia, duration of surgery, and duration from intubation to start of surgery (Table 2).

Of the 1280 (80 patients, 4 time points, 4 stress hormones) blood samples for stress hormones' assay, 143 samples were excluded from final analysis due to technical issues (such as insufficient amount of blood withdrawn in vial). The remaining 1137 samples were valid for final analysis.

The plasma concentrations of the stress hormones at the four event-related time points in both groups are presented in Table 3 and Figure 1. In both groups, the plasma levels of all the four stress hormones (ACTH, cortisol, epinephrine, and norepinephrine) decreased from Base to After-Maxi in other words, the plasma levels of the four stress hormones at Base were the highest and at After-Maxi were the lowest among the four event-related time points (Intu, Maxi, and After-Maxi compared with Base, $P<0.05$ ). No differences with respect to stress hormone levels at the event-related time points were found between the SPI group and the Control group $(P>0.05)$.

Correlations between SPI values and stress hormones (pooled data from both groups) at event-related time points are presented in Figure 2. For all four stress hormones, the absolute values of the correlation coefficient $(r)$ at time point Base were the lowest among the four event-related time points $(P<0.0001)$. At the time point Base, values of $r$ were less than 0.3 , indicating there was no meaningful correlation between SPI values and stress hormones at the time-point Base. Whereas at the other three time points (Intu, Maxi and After-Maxi), absolute values of $r$ were between 0.3 and 0.5 (except for the $r$ between SPI and epinephrine at the time-point of After-Maxi), indicating that SPI had 
TABLE 3: Stress hormones levels, mean Bp, heart rate, SPI, and BIS values at the different event-related time points.

\begin{tabular}{|c|c|c|c|c|c|c|c|c|c|}
\hline $\begin{array}{l}\text { Time } \\
\text { point }\end{array}$ & Group & $\begin{array}{c}\text { ACTH } \\
(\mathrm{pg} / \mathrm{mL})\end{array}$ & $\begin{array}{l}\text { Cortisol } \\
(\mathrm{pg} / \mathrm{mL})\end{array}$ & $\begin{array}{c}\text { Epinephrine } \\
(\mathrm{pg} / \mathrm{mL})\end{array}$ & $\begin{array}{c}\text { Norepinephrine } \\
(\mathrm{pg} / \mathrm{mL})\end{array}$ & $\begin{array}{c}\text { Mean } \\
(\mathrm{mmHg})\end{array}$ & $\begin{array}{c}\mathrm{HR} \\
\text { (beat/min) }\end{array}$ & SPI & BIS \\
\hline \multirow{2}{*}{ Base } & SSI & $23 \pm 14$ & $14 \pm 5$ & $38 \pm 18$ & $187 \pm 114$ & $94 \pm 14$ & $71 \pm 13$ & $53 \pm 11$ & $92 \pm 15$ \\
\hline & Control & $19 \pm 13$ & $13 \pm 6$ & $34 \pm 18$ & $252 \pm 172$ & $99 \pm 14$ & $70 \pm 12$ & $53 \pm 11$ & $97 \pm 2$ \\
\hline \multirow{2}{*}{ Intu } & SSI & $15 \pm 7^{* *}$ & $10 \pm 4^{* *}$ & $28 \pm 19$ & $129 \pm 69^{* *}$ & $94 \pm 20$ & $72 \pm 12$ & $51 \pm 13$ & $37 \pm 10^{* *}$ \\
\hline & Control & $16 \pm 9$ & $11 \pm 4^{*}$ & $26 \pm 9$ & $132 \pm 73^{* *}$ & $93 \pm 16$ & $75 \pm 15$ & $53 \pm 14$ & $37 \pm 13^{* *}$ \\
\hline \multirow{2}{*}{ Maxi } & SSI & $10 \pm 5^{* * \#}$ & $8 \pm 4^{* * \# \#}$ & $34 \pm 17$ & $83 \pm 50^{* * \# \#}$ & $74 \pm 10^{* * \# \#}$ & $59 \pm 10^{* * \# \#}$ & $43 \pm 12^{* * \# \#}$ & $39 \pm 11^{* *}$ \\
\hline & Control & $11 \pm 65^{* * \#}$ & $8 \pm 3^{* * \# \#}$ & $35 \pm 24^{\#}$ & $82 \pm 49^{* * \#}$ & $77 \pm 16^{* * \# \#}$ & $59 \pm 10^{* * \# \#}$ & $45 \pm 14^{* \#}$ & $38 \pm 9^{* *}$ \\
\hline \multirow{2}{*}{ After-Maxi } & SSI & $9 \pm 6^{* * \# \#}$ & $5 \pm 3^{* * \# \# \&}$ & $19 \pm 18^{* * \# \& \& \&}$ & $35 \pm 19^{* * \# \# \& \&}$ & $77 \pm 13^{* * \# \#}$ & $59 \pm 9^{* * \# \#}$ & $29 \pm 11^{* * \# \# \& \& \&}$ & $44 \pm 11^{* *}$ \\
\hline & Control & $9 \pm 5^{* * \# \#}$ & $6 \pm 4^{* * \# \#}$ & $19 \pm 10^{* * \& \&}$ & $36 \pm 18^{* * \# \# \&}$ & $82 \pm 15^{* * \# \#}$ & $58 \pm 10^{* * \# \#}$ & $31 \pm 13^{* * \# \# \& \&}$ & $40 \pm 7^{* *}$ \\
\hline
\end{tabular}

Values are mean \pm SD. Base $=$ baseline before anaesthesia; Intu = after tracheal intubation; Max = at maximum operative trauma which defined intraoperatively by the surgeon; After-Max = 15 min after the maximum operative trauma; SSI = the group in which remifentanil administration was guided by SSI; Control = the group in which remifentanil administration was guided by standard traditional inadequate anaesthesia criteria.

${ }^{*} P<0.05,{ }^{*} P<0.01$ compare with respective values at Base in the same group with one-way ANOVA followed by LSD test.

${ }^{\#} P<0.05,{ }^{\#} P<0.01$ compared with respective values at Intu in the same group with one-way ANOVA followed by LSD test.

${ }^{\&} P<0.05,{ }^{\& \&} P<0.01$ compared with respective values at Maxi in the same group with one-way ANOVA followed by LSD test.

No any differences were found at all four event-related timepoints between the SPI group and the Control group $(P>0.05)$.

a moderate or good correlation to stress hormones during general anaesthesia.

Regarding pooled data, SPI could indicate the predefined "specific level" (defined as the average of the data at the time point Base) of ACTH with an area under the curve of 0.85 $(P<0.0001)$, of cortisol with an area under the curve of $0.61(P=0.00299)$, of epinephrine with $0.59(P=0.0198)$, and norepinephrine with $0.62(P=0.0143)$. The optimal threshold value given by ROC analysis for SPI was an SPI $\geq 46$ to predict an ACTH of $\geq 15 \mathrm{pg} / \mathrm{mL}$ with a sensitivity of $81 \%$ and a specificity of $73 \%$, the threshold of SPI was $\geq 44$ to predict a cortisol of $\geq 12 \mu \mathrm{g} / \mathrm{dL}$ with a sensitivity of $66 \%$ and a specificity of $53 \%$, the threshold of SPI was $\geq 46$ to predict an epinephrine $\geq 25 \mathrm{pg} / \mathrm{mL}$ with a sensitivity of $59 \%$ and a specificity of $53 \%$, and the threshold of SPI was $\geq 44$ to predict a norepinephrine $\geq 220 \mathrm{pg} / \mathrm{mL}$ with a sensitivity of $64 \%$ and a specificity of $51 \%$.

\section{Discussion}

In the present study, we investigated the correlation between the surgical pleth index (SPI), a noninvasive variable derived from photoplethysmography which was developed originally for detecting stress or nociception level during general anaesthesia, with stress hormones in the circulating blood in a prospective, single-blinded, randomized study, and demonstrated that (1) SPI had no correlation with stress hormones at the time-point Base, whereas there was moderate-togood correlation with the stress hormones at the time-points Intu (after tracheal intubation), Max (at maximum surgical trauma defined intraoperatively by the attending surgeon), and After-Max (15 min after the maximum surgical trauma); (2) SPI could predict a specific level of ACTH with high sensitivity and specificity.

It is well known that measuring stress or nociception level during general anaesthesia is very challenging since there are no direct methods to measure it $[5,22]$. As a result, some unspecific autonomic reactions, such as blood pressure, heart rate, sweating, or tearing, are used traditionally for clinical evaluation of stress or nociception level during general anaesthesia, which has been proved unreliable with low specificity $[5,6]$. Clinical endpoints, such as movement in response to nociceptive stimulation, are commonly used as an indicator of inadequate analgesia [23] but are also unreliable and suppressed by muscle relaxants [24]. Some electroencephalographic (EEG-) derived variables, such as Entropy, especially the difference between state entropy (SE) and response entropy (RE), are proposed to be useful for evaluating the nociceptive component of anaesthesia $[17,25,26]$. Additionally, changes in skin conductivity and photoplethysmographic pulse wave amplitude or pulse wave reflex have been suggested as indicators of stress or nociception [27, 28]. However, the performance of these variables in general is disappointing $[29,30]$. Recently, surgical pleth index (SPI) [8], a multivariate index based on the sum of the photoplethysmographic pulse wave amplitude (PPGA) and the normalized heart beat interval (HBI), was developed for measuring the stress or nociception level during general anaesthesia. Several studies showed that the SPI had a negative correlation with remifentanil effectsite concentrations $\left(\mathrm{Ce}_{\mathrm{remi}}\right)$ and positive correlation with stimulus intensity during total i.v. anaesthesia with propofol; moreover, the SPI had a better performance in detecting nociceptive stimulation than state entropy (SE), response entropy (RE), heart rate, or PPGA, and the performance in reflecting the stress or nociception level was not influenced by the use of a $\beta$-blocker (Esmolol) [3, 9, 10, 22, 31]. However, for a newly developed measurement index such as SPI, evaluation of the performance in detecting the stress or nociception level during general anaesthesia should be as comprehensive as possible.

The stress response to surgery is characterized by an increased secretion of pituitary hormones which have secondary effects on hormone secretion from target organs [32]. Hence, ACTH, cortisol, epinephrine and norepinephrine serve as so-called stress hormones, and their blood levels 

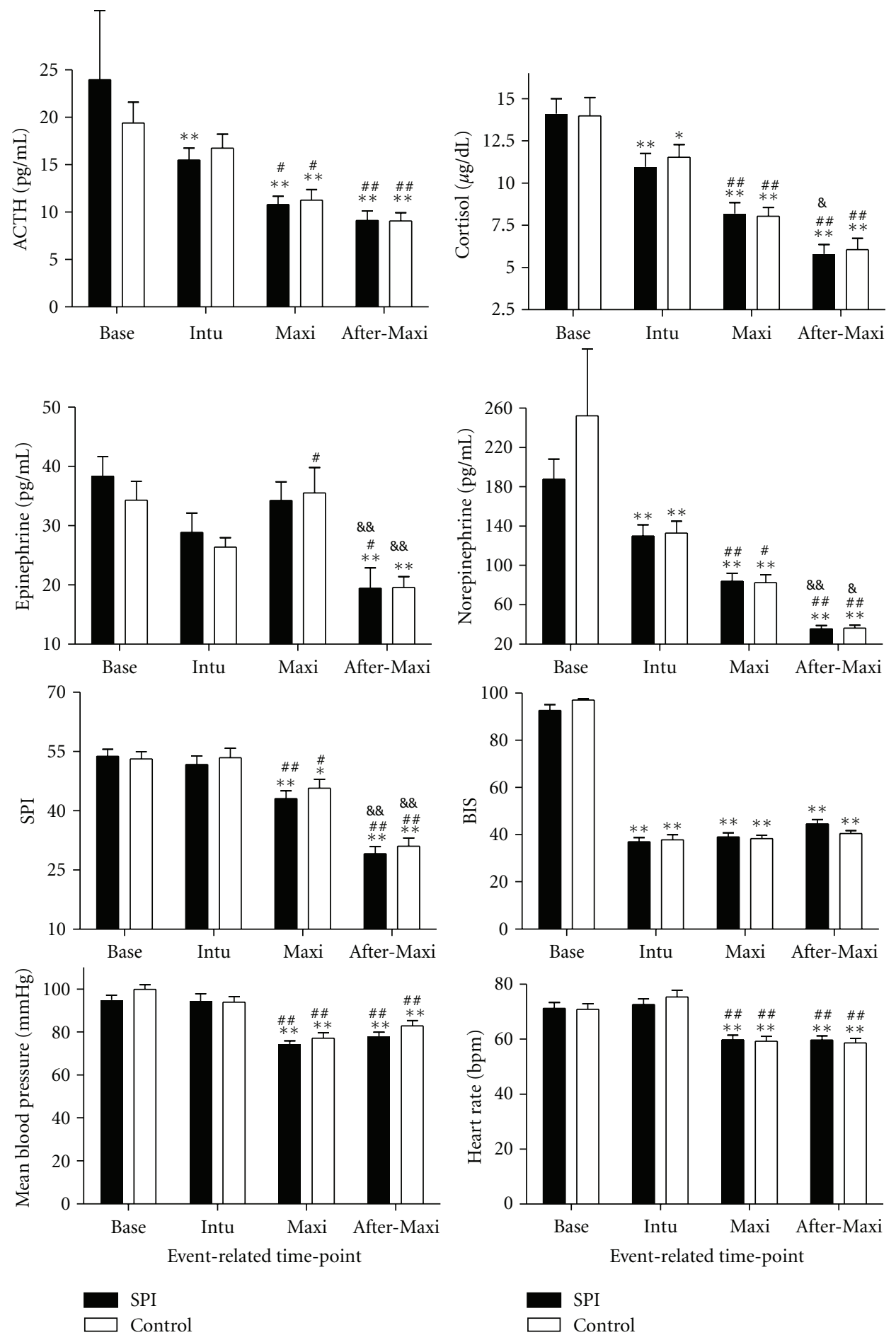

SPI

Control

FIGURE 1: Values are mean \pm SD. Base: baseline before anaesthesia; Intu: after tracheal intubation; Max: at maximum operative trauma which was defined intraoperatively by the surgeon; After-Max: 15 min after the maximum operative trauma; Mean: mean blood pressure; HR: heart rate; SPI: the group in which remifentanil administration was guided by SPI; Control: the group in which remifentanil administration was guided by standard traditional inadequate anaesthesia criteria. ${ }^{*} P<0.05,{ }^{*} P<0.01$ compared with respective values at Base in the same group with one-way ANOVA followed by LSD test. ${ }^{\#} P<0.05,{ }^{\# \#} P<0.01$ compared with respective values at Intu in the same group with one-way ANOVA followed by LSD test. ${ }^{\&} P<0.05$, \&\& $P<0.01$ compared with respective values at Maxi in the same group with one-way ANOVA followed by LSD test. No differences were found between the SPI group and the Control group at all four event-related time points $(P>0.05)$. 

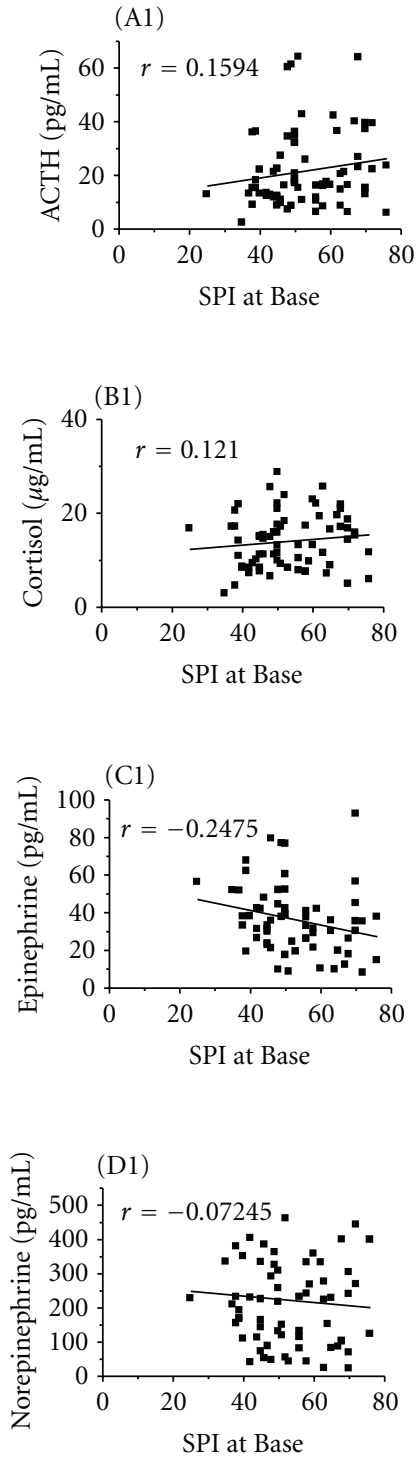

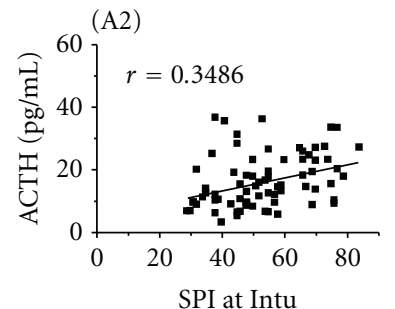

(a)
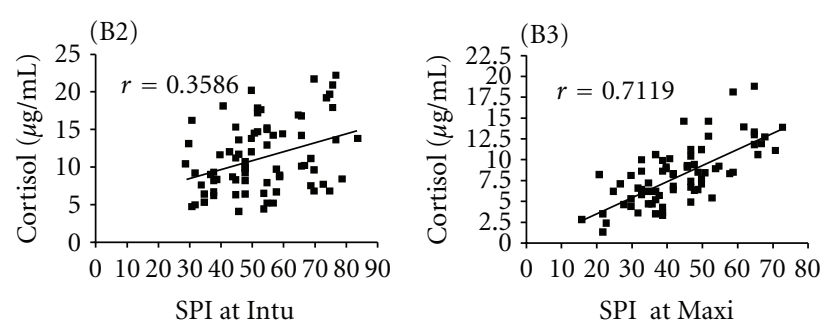

(b)
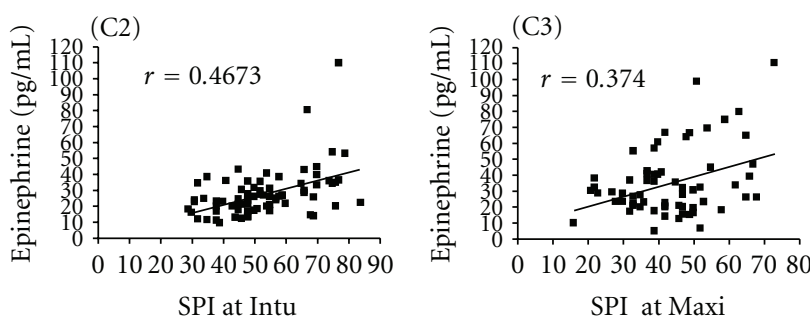

(c)
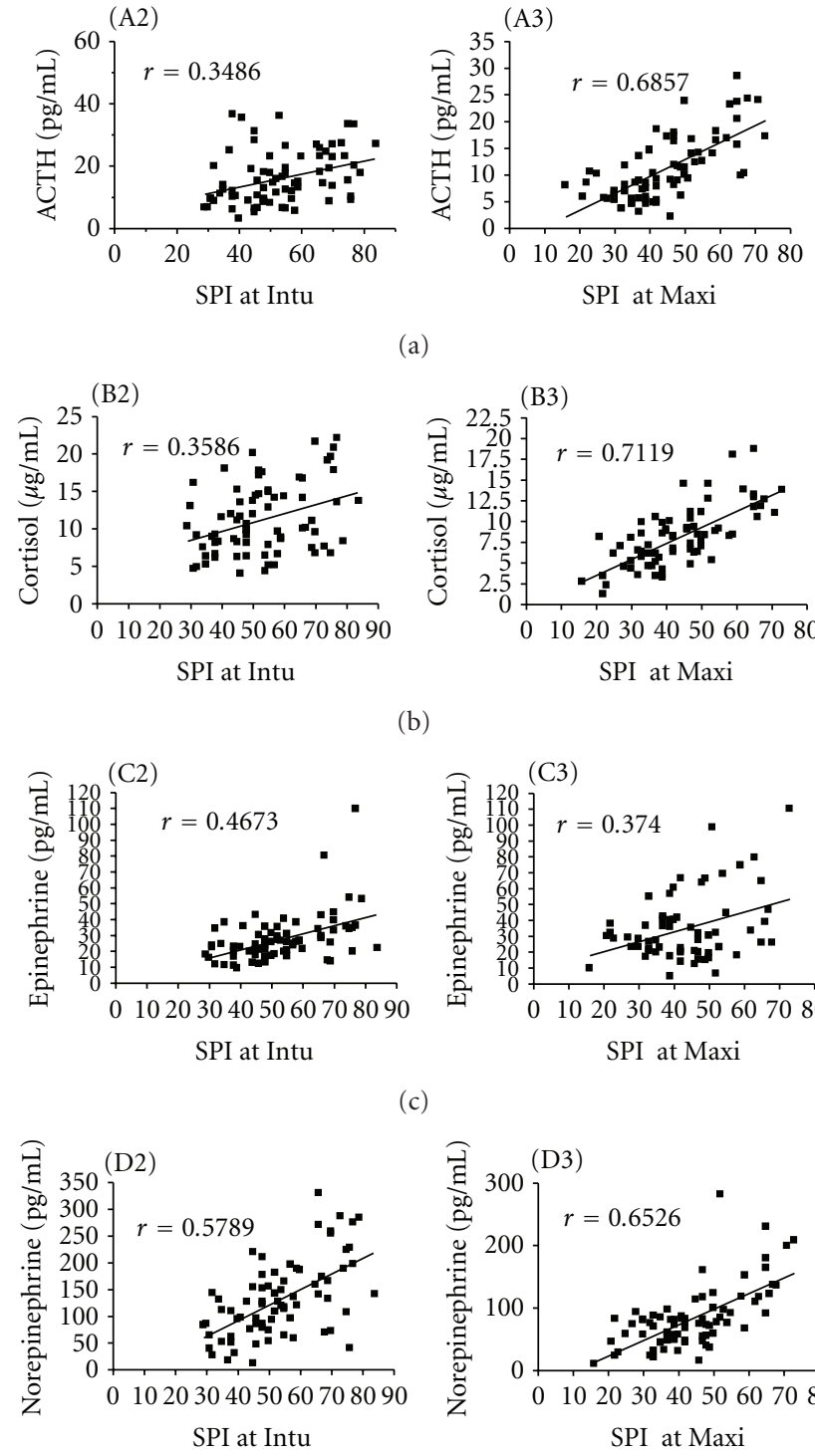
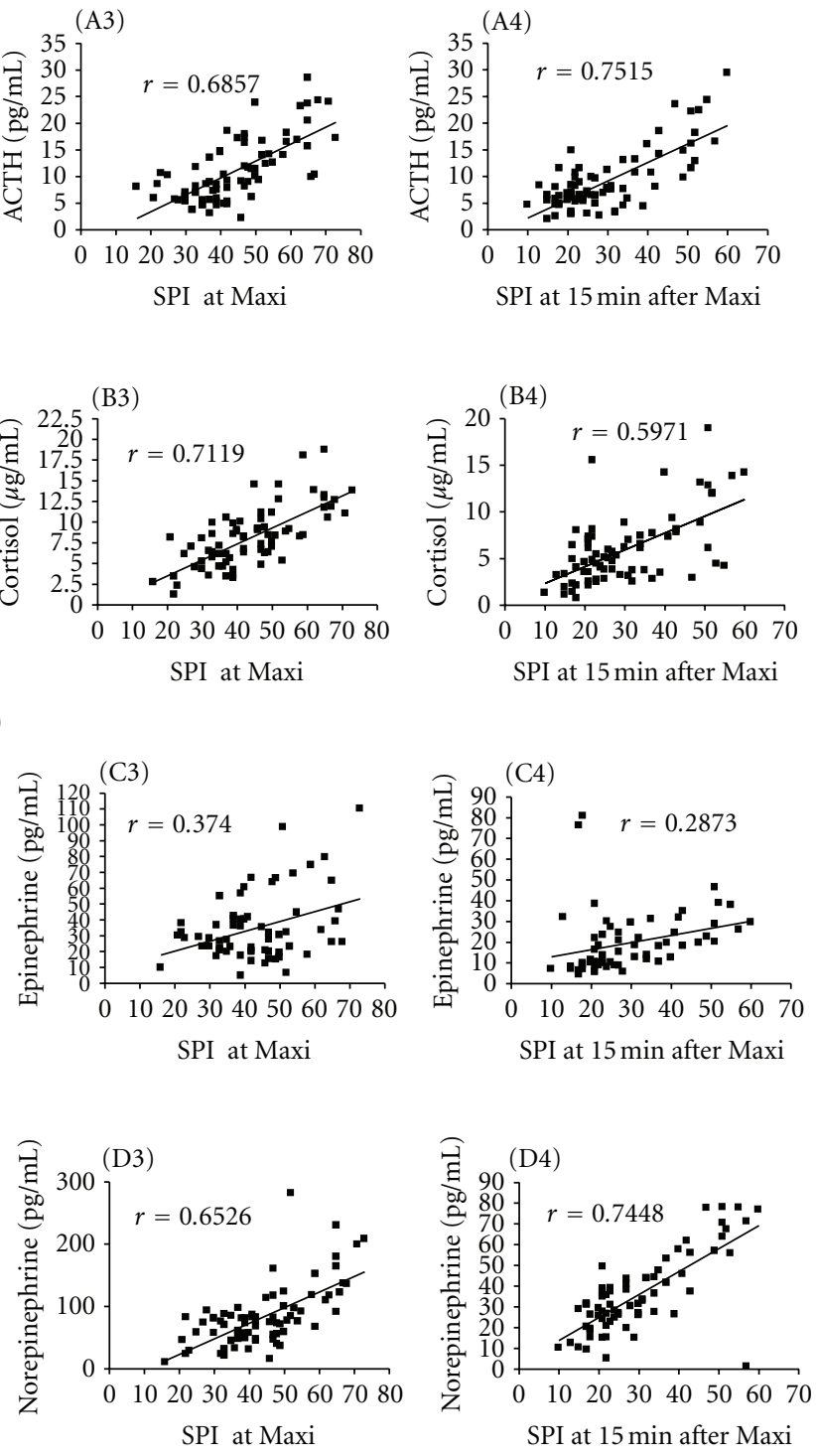

(d)

FIGURE 2: Linear regression analysis and correlation analysis between the SSI values and and stress hormones (ACTH, cortisol, epinephrine and norepinephrine). Bold line is regression line. Slopes of the above lines between time points are significantly different, $P<0.01$. ACTH: adrenocorticotropic hormone; SPI: surgical pleth index; Base: baseline before anaesthesia; Intu: after tracheal intubation; Max: at maximum operative trauma, defined intraoperatively by the surgeon; After-Max: $15 \mathrm{~min}$ after the maximum operative trauma.

were validated to correlate with intraoperative nociception and used for evaluating the stress or nociception level in surgery [14-16, 33]. It was also proven that excessive secretion of stress hormones was related to worse outcome [1]. In the present study, all four stress hormones were highest at "Base" when all patients were still conscious with some kind of mental stress despite premedication. Moreover, stress hormones were significantly higher at Max which was defined as the time of maximum surgical trauma indicated intraoperatively by the attending surgeon than at $15 \mathrm{~min}$ After-Max, indicating that stress hormones reflected well the stress level during general anaesthesia.

Interestingly, we found no correlation between stress hormones and SPI levels at Base, whereas there was a moderate-to-good correlation at the other three eventrelated time points. As reported, the SPI was developed by Huiku et al. [8] from anaesthetized patients aiming for detecting surgical stress during general anaesthesia. Patients at Base were awake, and may have suffered from preoperative anxiety consequently inducing a stress response, whereas during general anaesthesia patients were unconscious and psychogenic factors unlikely to interfere. Therefore, surgical induced nociception is suggested to be the main factor to elicit a stress response during general anaesthesia. This might be the reason of the discrepancy between correlation of stress hormones and SPI levels at different states of consciousness. Consequently it might be suggested that SPI only works well in anaesthetized patients. 
The difference with respect to the magnitude of correlation of the stress hormones with the SPI between the four stress hormones may be explained as follows (1) It is well known that a subject's response to stress, such as surgical stimulation, is characterized by an activation of the hypothalamic-pituitary-adrenal axis, triggering release of ACTH which then stimulates secretion of cortisol from the adrenal cortex. Moreover, stress-induced activation of the hypothalamic-pituitary-adrenal axis HPA axis also initiates sympathetic nervous system production of the catecholamines epinephrine and norepinephrine [34, 35]. The secretion of these stress hormones has a timing sequence and hence at different time point the levels of the stress hormones might be different. In the present study, blood samples for the stress hormones assay were collected between $30 \mathrm{~s}$ to $60 \mathrm{~s}$ after the event-related time point. This may explain why some stress hormones were already at peak levels whereas other hormones lagged behind. (2) It was shown that responses of stress hormones to surgical stimulation are different [36-38]. Catecholamines such as epinephrine and norepinephrine were reported to be relatively unaffected by the choice of the main anaesthetic drug, and surgical stress caused a similar variation of catecholamines with different inhaled anaesthetics whereas an increase of $\mathrm{ACTH}$ and subsequent increase of cortisol are known to correlate well with the severity of surgical stress [37]. Furuya et al. found that plasma concentrations of epinephrine and norepinephrine were within the normal range during surgery whereas plasma concentrations of ACTH and cortisol increased $30 \mathrm{~min}$ and 60 min after incision. Further, time to peak differed between hormones. This points to the fact that different stress hormones may have different characteristics with respect to their release after nociceptive stimulation.

Some limitations of the present study should be noted. First, the choice of time points for blood withdrawal may be argued. However, the time points we choose are event related, which referred to nociceptive stimuli level during the course of general anaesthesia. Second, the exact distance between the respective event and blood withdrawal is difficult to define. In our study, we decided to collect blood samples one minute after the event to allow for the release of stress hormones in the circulating blood, while SPI values were noted 15 seconds after the event to allow for the newest data to be displayed on the screen. Third, ENT surgery has lower surgical stimulus relative to other large sugery, which might be the reason that all stress hormones (except epinephrine) were low in the present study. Hence, more styles of surgery are needed to be included in further studies

In conclusion, the SPI showed moderate correlation to the stress hormones (ACTH, cortisol, epinephrine, and norepinephrine) during general anaesthesia, but no correlation during consciousness. Furthermore, SPI values were able to predict ACTH values with high sensitivity and specificity.

\section{Conflict of Interests}

Dr. B. Bein has received honoraria for consulting and giving lectures from GE Healthcare, the manufacturer of the SPI.
The other authors declare that they have no conflict of interest.

\section{Acknowledgments}

The authors are grateful to GE Healthcare, Helsinki, Finland, for providing the SPI module. The authors are indebted to Werner Jeckstroem, M.D. (Staff Member, Department of Anaesthesiology and Intensive Care Medicine, University Hospital Schleswig-Holstein, Campus Kiel, Germany), for his enthusiastic support.

\section{References}

[1] S. D. Parker, M. J. Breslow, S. M. Frank et al., "Catecholamine and cortisol responses to lower extremity revascularization: correlation with outcome variables," Critical Care Medicine, vol. 23, no. 12, pp. 1954-1961, 1995.

[2] P. S. Myles, J. O. Hunt, H. Fletcher et al., "Remifentanil, fentanyl, and cardiac surgery: a double-blinded, randomized, controlled trial of costs and outcomes," Anesthesia and Analgesia, vol. 95, no. 4, pp. 805-812, 2002.

[3] M. M. R. F. Struys, C. Vanpeteghem, M. Huiku, K. Uutela, N. B. K. Blyaert, and E. P. Mortier, "Changes in a surgical stress index in response to standardized pain stimuli during propofol-remifentanil infusion," British Journal of Anaesthesia, vol. 99, no. 3, pp. 359-367, 2007.

[4] W. Buhre and R. Rossaint, "Perioperative management and monitoring in anaesthesia," Lancet, vol. 362, no. 9398, pp. 1839-1846, 2003.

[5] J. Bruhn, P. S. Myles, R. Sneyd, and M. M. R. F. Struys, "Depth of anaesthesia monitoring: what's available, what's validated and what's next?" British Journal of Anaesthesia, vol. 97, no. 1, pp. 85-94, 2006.

[6] T. Heier and P. A. Steen, "Assessment of anaesthesia depth," Acta Anaesthesiologica Scandinavica, vol. 40, no. 9, pp. 10871100, 1996.

[7] M. Rantanen, A. Yli-Hankala, M. van Gils et al., "Novel multiparameter approach for measurement of nociception at skin incision during general anaesthesia," British Journal of Anaesthesia, vol. 96, no. 3, pp. 367-376, 2006.

[8] M. Huiku, K. Uutela, M. van Gils et al., "Assessment of surgical stress during general anaesthesia," British Journal of Anaesthesia, vol. 98, no. 4, pp. 447-455, 2007.

[9] J. Wennervirta, M. Hynynen, A. M. Koivusalo, K. Uutela, M. Huiku, and A. Vakkuri, "Surgical stress index as a measure of nociception/antinociception balance during general anesthesia," Acta Anaesthesiologica Scandinavica, vol. 52, no. 8, pp. 1038-1045, 2008.

[10] M. Gruenewald, P. Meybohm, C. Ilies et al., "Influence of different remifentanil concentrations on the performance of the surgical stress index to detect a standardized painful stimulus during sevoflurane anaesthesia," British Journal of Anaesthesia, vol. 103, no. 4, pp. 586-593, 2009.

[11] W. D. Smith, R. C. Dutton, and N. T. Smith, "Measuring the performance of anesthetic depth indicators," Anesthesiology, vol. 84, no. 1, pp. 38-51, 1996.

[12] W. D. Smith, R. C. Dutton, and N. T. Smith, "A measure of association for assessing prediction accuracy that is a generalization of non-parametric roc area," Statistics in Medicine, vol. 15, pp. 1199-1215, 1996.

[13] X. Chen, C. Thee, M. Gruenewald et al., "Comparison of surgical stress index-guided analgesia with standard clinical 
practice during routine general anesthesia: a pilot study," Anesthesiology, vol. 112, no. 5, pp. 1175-1183, 2010.

[14] T. Schricker, F. Carli, M. Schreiber et al., "Propofol/sufentanil anesthesia suppresses the metabolic and endocrine response during, not after, lower abdominal surgery," Anesthesia and Analgesia, vol. 90, no. 2, pp. 450-455, 2000.

[15] M. F. Roizen, R. W. Horrigan, and B. M. Frazer, "Anesthetic doses blocking adrenergic (stress) and cardiovascular responses to incision-MAC BAR," Anesthesiology, vol. 54, no. 5, pp. 390-398, 1981.

[16] T. Ledowski, B. Bein, R. Hanss et al., "Neuroendocrine stress response and heart rate variability: a comparison of total intravenous versus balanced anesthesia," Anesthesia and Analgesia, vol. 101, no. 6, pp. 1700-1705, 2005.

[17] M. Gruenewald, J. Zhou, N. Schloemerkemper et al., "MEntropy guidance vs standard practice during propofolremifentanil anaesthesia: a randomised controlled trial," Anaesthesia, vol. 62, no. 12, pp. 1224-1229, 2007.

[18] S. Kreuer, A. Biedler, R. Larsen, S. Altmann, and W. Wilhelm, "Narcotrend monitoring allows faster emergence and a reduction of drug consumption in propofol-remifentanil anesthesia," Anesthesiology, vol. 99, no. 1, pp. 34-41, 2003.

[19] M. A. Garrioch and W. Fitch, "Anaesthesia for carotid artery surgery," British Journal of Anaesthesia, vol. 71, no. 4, pp. 569579, 1993.

[20] T. W. Schnider, C. F. Minto, P. L. Gambus et al., "The influence of method of administration and covariates on the pharmacokinetics of propofol in adult volunteers," Anesthesiology, vol. 88, no. 5, pp. 1170-1182, 1998.

[21] C. F. Minto, T. W. Schnider, and S. L. Shafer, "Pharmacokinetics and pharmacodynamics of remifentanil II. Model application," Anesthesiology, vol. 86, no. 1, pp. 21-33, 1997.

[22] S. Mustola, T. Parkkari, K. Uutela, M. Huiku, M. Kymäläinen, and J. Toivonen, "Performance of surgical stress index during sevoflurane-fentanyl and isoflurane-fentanyl anesthesia," Anesthesiology Research and Practice, vol. 2010, Article ID 810721, 5 pages, 2010.

[23] A. M. Zbinden, M. Maggiorini, S. Petersen-Felix et al., "Anesthetic depth defined using multiple noxious stimuli during isoflurane/oxygen anesthesia: I. Motor reactions," Anesthesiology, vol. 80, no. 2, pp. 253-260, 1994.

[24] R. H. Sandin, G. Enlund, P. Samuelsson, and C. Lennmarken, "Awareness during anaesthesia: a prospective case study," Lancet, vol. 355, no. 9205, pp. 707-711, 2000.

[25] B. Bein, "Entropy," Best Practice \& Research Clinical Anaesthesiology, vol. 20, pp. 101-109, 2006.

[26] I. Takamatsu, M. Ozaki, and T. Kazama, "Entropy indices vs the bispectral index for estimating nociception during sevoflurane anaesthesia," British Journal of Anaesthesia, vol. 96, no. 5, pp. 620-626, 2006.

[27] H. Storm, K. Myre, M. Rostrup, O. Stokland, M. D. Lien, and J. C. Ræder, "Skin conductance correlates with perioperative stress," Acta Anaesthesiologica Scandinavica, vol. 46, no. 7, pp. 887-895, 2002.

[28] E. R. J. Seitsonen, I. K. J. Korhonen, M. J. Van Gils et al., "EEG spectral entropy, heart rate, photoplethysmography and motor responses to skin incision during sevoflurane anaesthesia," Acta Anaesthesiologica Scandinavica, vol. 49, no. 3, pp. 284292, 2005.

[29] B. Guignard, "Monitoring analgesia," Best Practice and Research: Clinical Anaesthesiology, vol. 20, no. 1, pp. 161-180, 2006.

[30] T. Nishiyama, "Recent advance in patient monitoring," Korean Journal of Anesthesiology, vol. 59, no. 3, pp. 144-159, 2010.
[31] J. Ahonen, R. Jokela, K. Uutela, and M. Huiku, "Surgical stress index reflects surgical stress in gynaecological laparoscopic day-case surgery," British Journal of Anaesthesia, vol. 98, no. 4, pp. 456-461, 2007.

[32] J. Desborough, "Endocrine response to surgery," in Anaesthesia Review, L. Kaufman, Ed., pp. 131-148, Churchill Livingstone, Edinburgh, UK, 1993.

[33] J. P. Desborough, "The stress response to trauma and surgery," British Journal of Anaesthesia, vol. 85, no. 1, pp. 109-117, 2000.

[34] L. J. Crofford, E. A. Young, N. C. Engleberg et al., "Basal circadian and pulsatile ACTH and cortisol secretion in patients with fibromyalgia and/or chronic fatigue syndrome," Brain, Behavior, and Immunity, vol. 18, no. 4, pp. 314-325, 2004.

[35] J. J. Haddad, N. E. Saadé, and B. Safieh-Garabedian, "Cytokines and neuro-immune-endocrine interactions: a role for the hypothalamic-pituitary-adrenal revolving axis," Journal of Neuroimmunology, vol. 133, no. 1-2, pp. 1-19, 2002.

[36] T. Nishiyama, K. Yamashita, and T. Yokoyama, "Stress hormone changes in general anesthesia of long duration: isoflurane-nitrous oxide vs sevoflurane-nitrous oxide anesthesia," Journal of Clinical Anesthesia, vol. 17, no. 8, pp. 586-591, 2005.

[37] C. Traynor and G. M. Hall, "Endocrine and metabolic changes during surgery: anaesthetic implications," British Journal of Anaesthesia, vol. 53, no. 2, pp. 153-160, 1981.

[38] J. B. Halter, A. E. Pflug, and D. Porte, "Mechanism of plasma catecholamine increases during surgical stress in man," Journal of Clinical Endocrinology and Metabolism, vol. 45, no. 5, pp. 936-944, 1977. 


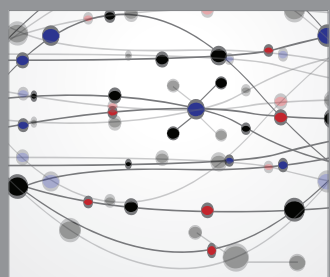

The Scientific World Journal
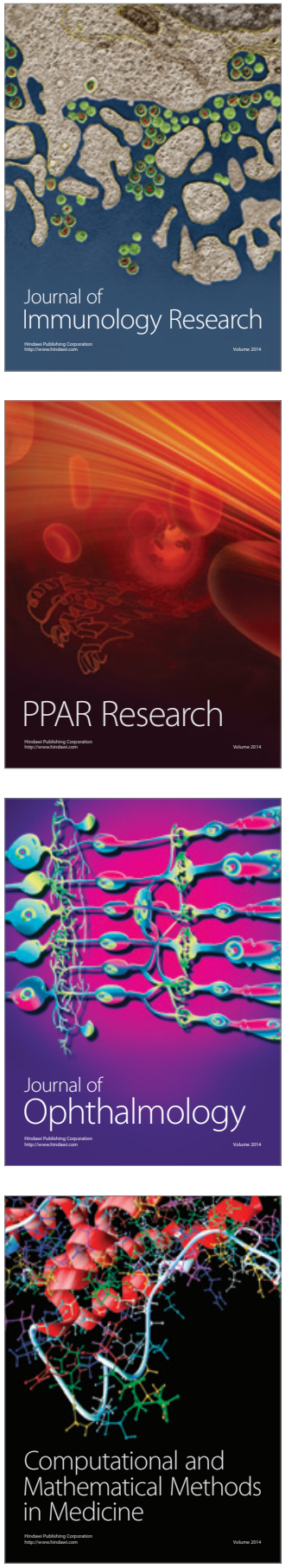

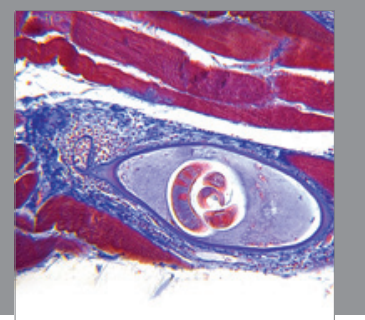

Gastroenterology

Research and Practice
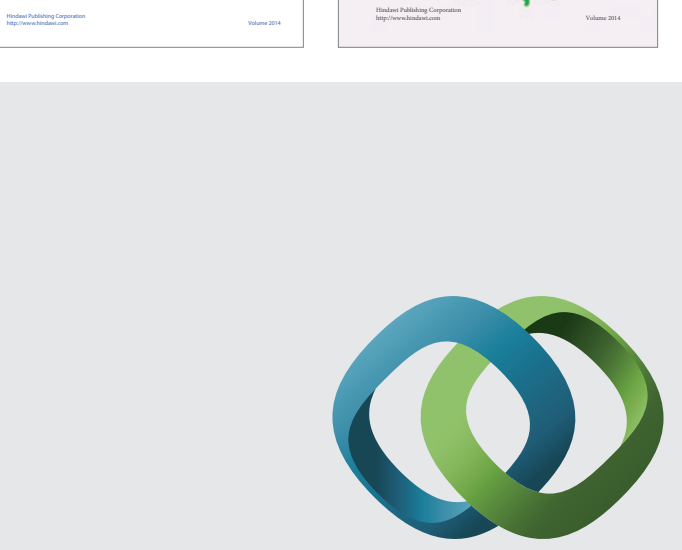

\section{Hindawi}

Submit your manuscripts at

http://www.hindawi.com
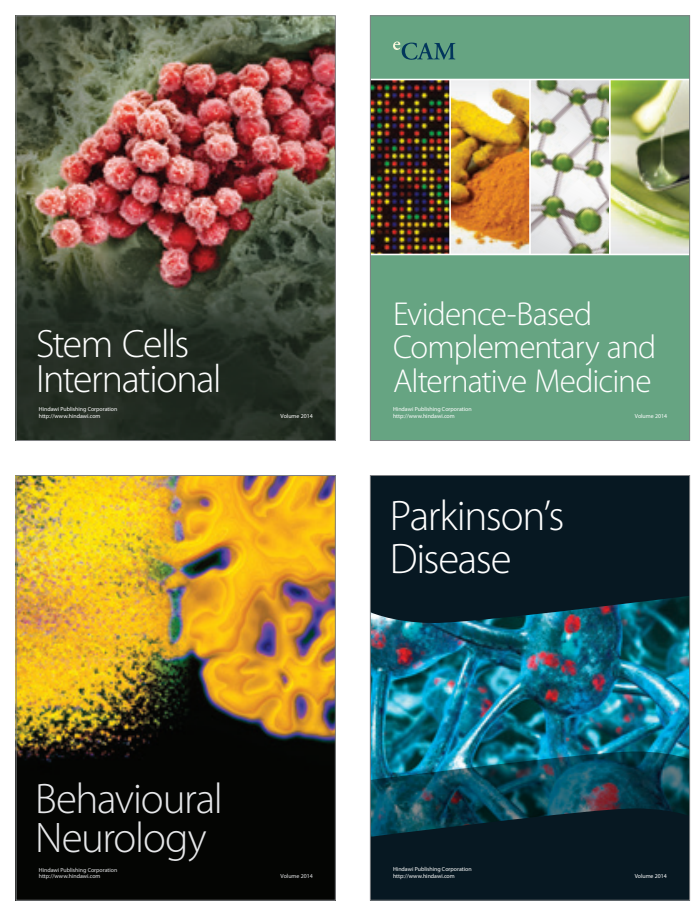

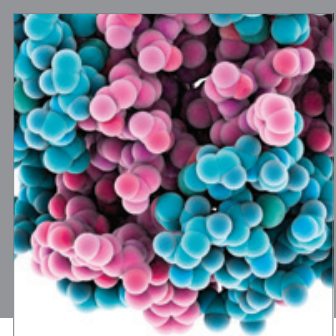

Journal of
Diabetes Research

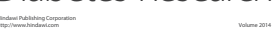

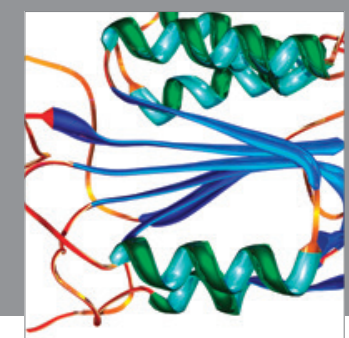

Disease Markers
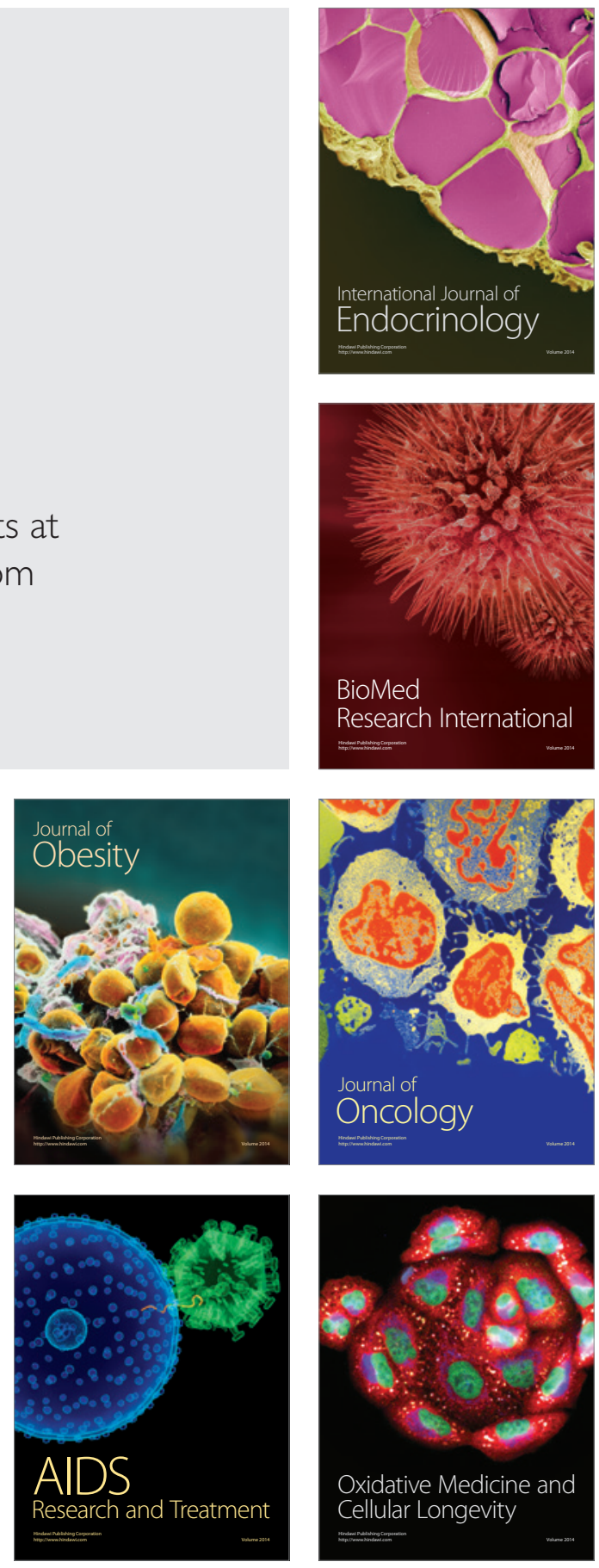